\section{Livestock-associated MRSA: innocent or serious health threat?}
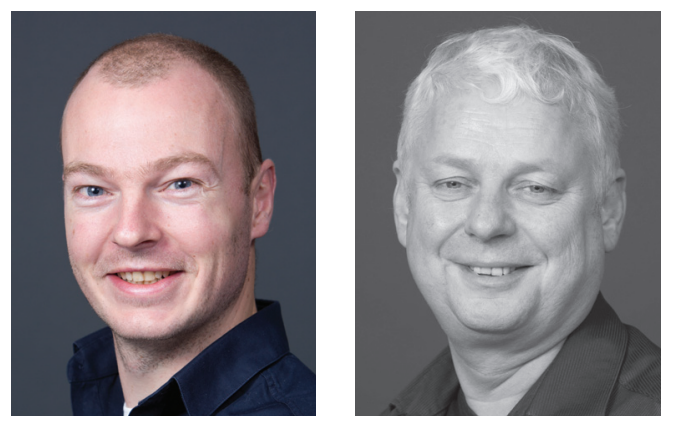

“...livestock-associated MRSA

may pose a serious health

threat and might potentially

lead to spread in the

community without the

involvement of the animal

reservoir."

Thijs Bosch ${ }^{*, 1} \&$ Leo M Schouls ${ }^{1}$

\section{MRSA from the animal reservoir}

In 2003, a novel MRSA variant associated with pigs, emerged in The Netherlands [1]. Soon after the first publication, other reports appeared describing the presence of this MRSA variant in numerous countries worldwide and in other animals associated with husbandry, resulting in the term livestock-associated MRSA (LA-MRSA) [2]. In The Netherlands, LA-MRSA prevalence in livestock animals such as pigs and veals is high [3,4], consequently the major risk factor for acquiring LA-MRSA is having contact with livestock [5]. Therefore, contact with livestock was added to the guidelines for the Dutch MRSA 'search \& destroy' policy in 2006 and 2007. As a result, the number of LA-MRSA isolated from humans has increased considerably and currently, around $30 \%$ of all MRSA isolates submitted for typing in the Dutch MRSA surveillance program are LA-MRSA.

Although MRSA isolated from livestock belongs to several clonal complexes (CCs), identified by multilocus sequence typing, the vast majority of LA-MRSA belong to CC398 [6]. LA-MRSA has distinct genotypic and phenotypic features that separate it from other MRSA variants. Examples are coresistance to certain antimicrobials used in the animal production, for example, tetracycline and the near absence of virulence factors such as Panton-Valentine leucocidin and enterotoxins $[7,8]$.

\section{Origin of LA-MRSA}

Molecular typing techniques, such as pulsed field gel electrophoresis, multilocus sequence typing, spa-typing and MLVA have been used to characterize LA-MRSA, but their discriminatory power appears too limited for this genotypic homogeneous MRSA clade. This has hampered studies on the origin and its ability to transmit between and persist in humans, but the introduction of new technologies such as next-generation sequencing (NGS) and whole-genome mapping have eliminated this limitation.

\section{KEYWORDS}

- infection - livestock-associated MRSA $\bullet$ persistence $\bullet$ transmission 
Using NGS, Price et al. analyzed 89 isolates and concluded that LA-MRSA likely originates from a human origin as methicillin-sensitive Staphylococcus aureus (MSSA). They theorized that the recent emergence of LA-MRSA can be seen as a reintroduction of the MSSA that had acquired methicillin-resistance into the original host [9]. However, other studies utilizing NGS suggested an independent human-specific lineage exists that differs from animal-related lineages in the spectrum of mobile genetic elements, such as bacteriophages [10-12].

Transmission and persistence of LA-MRSA One of the controversies regarding LA-MRSA is its ability to transmit between and persist in humans. Some reports have shown that transmission of LA-MRSA between humans is less likely to occur compared with other MRSA variants, particularly in the nosocomial setting [13,14]. In a recent study, transmissibility of LA-MRSA in Dutch hospitals was calculated to be 4.4-times lower than non-LA-MRSA and the authors suggest that preemptive isolation of patients carrying LA-MRSA may not be necessary [15] However, in this study, there were big differences between transmissibility of the various spa-types. For spa-type t008, the most abundant non-LAMRSA in both the study and in the Dutch MRSA surveillance, no transmission was found. We believe this shows that comparisons of transmissibility of different MRSA lineages should be made at the genotype level, and not at the group level such as MRSA versus LA-MRSA. Also, we are convinced that transmission studies can only be performed using high-resolution typing techniques. We were able to show, by characterizing isolates using high-resolution genotyping, that presumed LA-MRSA transmissions were actually not transmission events [16].

There is generally believed that LA-MRSA is incapable to colonize and subsequently persist in humans. Indeed, Graveland et al. showed that only $7 \%$ of the veal farmers persistently carried LA-MRSA [17]. By contrast, a study among pig farmers found ongoing carriage of LA-MRSA in 59\% of the farmers despite an absence of animal contact during holidays [18]. In a recent study among livestock veterinarians, we showed that nearly $30 \%$ of the veterinarians and their household members, who did not have direct contact with livestock, persistently carried LA-MRSA with carriage periods ranging from 4 to 14 months [16].

\section{MRSA CC398 infections}

Although the ability of LA-MRSA to cause disease in humans has also been a subject of debate, a wide array of infections in humans due to LA-MRSA has been reported, varying from wound infections to bacteremia [19]. An increase in the CC398 prevalence in bloodstream infections was also observed, but most of these isolates were methicillin susceptible and originated from patients lacking livestock contact [20]. In The Netherlands, bloodstream infections caused by MRSA are rare. However, $20 \%$ of the MRSA isolated from blood are LA-MRSA, although the distribution may be slightly skewed as $30 \%$ of all Dutch surveillance isolates are LA-MRSA. Yet, comparison of the group non-LA-MRSA and LA-MRSA as a whole shows that non-LA-MRSA is two- to three-times more frequently associated with bloodstream infections than LA-MRSA. Recently, the Danish Statens Serum Institute reported four case histories of individuals that died from sepsis caused by LA-MRSA (SSI, EpiNews No. 24a -2014). Remarkably, none of these patients had any contact to pig farming.

In conclusion, we believe that LA-MRSA is a successful colonizer in a broad range of hosts, including man. There is ample evidence that LA-MRSA is capable of transmitting between and persisting in humans. Studies on transmissibility are scarce and those performed may need to be re-evaluated. The enormous number of carriage isolates may have obscured the potential of LA-MRSA to spread and cause disease. However, at least in the Dutch MRSA surveillance, bloodstream infections caused by LA-MRSA are found in similar proportions as those caused by non-LA-MRSA. These characteristics imply LA-MRSA may pose a serious health threat and might potentially lead to spread in the community without the involvement of the animal reservoir. This is supported by the four fatal cases in Denmark where patients had no contact with livestock and a recent study in The Netherlands showing that $26 \%$ of the MRSAs with an unknown origin belonged to CC398 [21]. We believe that, until proven otherwise, LA-MRSA remains as pathogenic as most non-LA-MRSA and should be treated as such.

\section{Financial \& competing interests disclosure}

The authors have no relevant affiliations or financial involvement with any organization or entity with a financial interest in or financial conflict with the subject matter or materials discussed in the manuscript. This includes employment, 
consultancies, honoraria, stock ownership or options, expert testimony, grants or patents received or pending, or royalties.

\section{References}

1 Voss A, Loeffen F, Bakker J, Klaassen C, Wulf M. Methicillin-resistant Staphylococcus aureus in pig farming. Emerg. Infect. Dis. 11(12), 1965-1966 (2005).

2 Smith TC, Pearson N. The emergence of Staphylococcus aureus ST398. Vector Borne Zoonotic Dis. 11(4), 327-339 (2011).

3 de Neeling AJ, van den Broek MJ, Spalburg EC et al. High prevalence of methicillin resistant Staphylococcus aureus in pigs. Vet. Microbiol. 122(3-4), 366-372 (2007).

4 Graveland H, Wagenaar JA, Heesterbeek H et al. Methicillin resistant Staphylococcus aureus ST398 in veal calf farming: human MRSA carriage related with animal antimicrobial usage and farm hygiene. PLoS ONE 5(6), e10990 (2010).

5 Van Den Broek IVF, Van Cleef BAGL, Haenen A et al. Methicillin-resistant Staphylococcus aureus in people living and working in pig farms. Epidemiol Infect. 137(5), 700-708 (2009).

6 Huijsdens XW, van Dijke BJ, Spalburg E et al. Community-acquired MRSA and pig-farming. Ann. Clin. Microbiol. Antimicrob. 5, 26 (2006).

7 Argudin MA, Tenhagen BA, Fetsch A et al. Virulence and resistance determinants of German Staphylococcus aureus ST398 isolates from nonhuman sources. Appl. Environ. Microbiol. 77(9), 3052-3060 (2011).

8 Hallin M, De Mendonca R, Denis O et al. Diversity of accessory genome of human and

No writing assistance was utilized in the production of this manuscript.

livestock-associated ST398 methicillin resistant Staphylococcus aureus strains. Infect. Genet. Evol. 11(2), 290-299 (2011).

9 Price LB, Stegger M, Hasman H et al. Staphylococcus aureus CC398: host adaptation and emergence of methicillin resistance in livestock. mBio 3(1), e00305-e00311 (2012).

10 McCarthy AJ, van Wamel W, Vandendriessche $S$ et al. Staphylococcus aureus CC398 clade associated with human-tohuman transmission. Appl. Environ. Microbiol. 78(24), 8845-8848 (2012).

11 Uhlemann AC, Porcella SF, Trivedi S et al. Identification of a highly transmissible animal-independent Staphylococcus aureus ST398 clone with distinct genomic and cell adhesion properties. mBio 3(2), e00027-12 (2012).

12 Ward MJ, Gibbons CL, McAdam PR et al. Time-scaled evolutionary analysis of the transmission and antibiotic resistance dynamics of Staphylococcus aureus CC398. Appl. Environ. Microbiol. pii: AEM.01777-14 (2014) (Epub ahead of print).

13 Bootsma MC, Wassenberg MW, Trapman P, Bonten MJ. The nosocomial transmission rate of animal-associated ST398 meticillinresistant Staphylococcus aureus. J. R. Soc. Interface 8(57), 578-584 (2011).

14 Wassenberg MW, Bootsma MC, Troelstra A, Kluytmans JA, Bonten MJ. Transmissibility of livestock-associated methicillin-resistant Staphylococcus aureus (ST398) in Dutch hospitals. Clin. Microbiol. Infect. 17(2), 316-319 (2011).
15 Hetem DJ, Bootsma MC, Troelstra A, Bonten MJ. Transmissibility of livestock-associated methicillin-resistant Staphylococcus aureus. Emerg. Infect. Dis. 19(11), 1797-1802 (2013).

16 Bosch T, Verkade E, van Luit $\mathrm{M}$ et al. Transmission and persistence of livestockassociated MRSA among veterinarians and their household members. Appl. Environ. Microbiol. 81(1), 124-129 (2015).

17 Graveland H, Wagenaar JA, Bergs K, Heesterbeek H, Heederik D. Persistence of livestock associated MRSA CC398 in humans is dependent on intensity of animal contact. PLoS ONE 6(2), el6830 (2011).

18 Kock R, Loth B, Koksal M et al. Persistence of nasal colonization with livestock-associated methicillin-resistant Staphylococcus aureus in pig farmers after holidays from pig exposure. Appl. Environ. Microbiol. 78(11), 4046-4047 (2012).

19 van Belkum A, Melles DC, Peeters JK et al. Methicillin-resistant and -susceptible Staphylococcus aureus sequence type 398 in pigs and humans. Emerg. Infect. Dis. 14(3), 479-483 (2008).

20 Valentin-Domelier AS, Girard M, Bertrand X et al. Methicillin-susceptible ST398 Staphylococcus aureus responsible for bloodstream infections: an emerging human-adapted subclone? PLoS ONE 6(12), e28369 (2011).

21 Lekkerkerk WS, van de Sande-Bruinsma N, van der Sande MA et al. Emergence of MRSA of unknown origin in The Netherlands. Clin. Microbiol. Infect. 18(7), 656-661 (2012). 